\title{
Lokaltherapie
}

\section{Suche Lösung für die Stomatitis aphthosa}

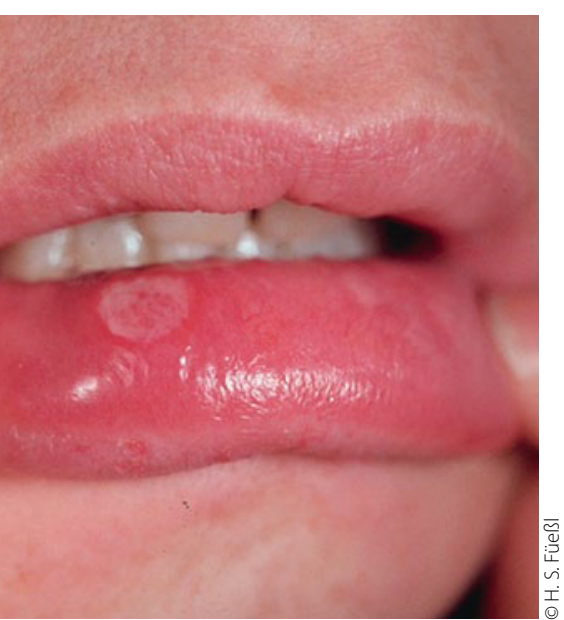

Stomatitis aphthosa: Lokaltherapeutikum gesucht.
Frage von Dr. med. N. N.

Neben der pflegenden Lokaltherapie der Stomatitis aphthosa z. B. mit PanthenolLösung gab es früher auch eine HervirosLösung mit Tetracain zur anästhesierenden Lokaltherapie. Diese scheint jetzt nicht mehr erhältlich zu sein. Die Applikation einer Lösung erscheint mir jedoch viel besser im Vergleich zu Gels, zumal bei ausgeprägtem Befund die Effloreszenzen bei Kontakt sofort bluten. An ein Einmassieren ist nicht zu denken. Was können Sie zur Lokaltherapie empfehlen? Gibt es geeignete Rezepturen?

Antwort von Dr. med. R. Kardoff, Wesel: Seit Jahren mache ich gute Erfahrungen mit einer 1:1:1-Lösung aus Hexoral,
Xylocain und Panthenol, die in unserer Klinikapotheke zubereitet wird. Konkret: Hexoral 0,1\% 6,66g (entspricht Hexetidin 6,66 mg), Xylocain Viscös $2 \%$ 6,66g (entspricht Lidocain- $\mathrm{HCl}$ 133,2 mg), Panthenol Lösung 6,66g (entspricht Dexpanthenol $333 \mathrm{mg}$ ). Das ergibt 20 g einer leicht viskösen Lösung, die in eine lichtschützende Glasflasche mit Tropfpipette kommt.

- Dr. med. Rüdiger Kardorff, Oberarzt, Facharzt für Kinderheilkunde - Kindergastroenterologie, Klinik für Kinder- und Jugendmedizin, Marien-Hospital Wesel gGmbH, PastorJanßen-Straße 8-38, D-46483 Wesel

\section{Arzt-Patienten-Kommunikation}

\section{Mit Nörglern kann ich nicht umgehen}

Frage von A. N.

In meinem Praxisalltag kommt es häufig vor, dass mir fordernde Patienten zu schaffen machen. Ich kann mich nicht abgrenzen und die notwendige Distanz finden, um mit dem nörgelnden, unzufriedenen Tonfall richtig umzugehen. Was können Sie mir raten?

Antwort von Dr. med. A.-J. Michel, München:

Das Lebensgefühl der Individuen ist mannigfaltig und nicht immer leicht $\mathrm{zu}$ erfassen. Krankheit erschwert vielen Menschen den Zugang zu anderen und engt die Vorstellungskraft über das, was außerhalb des Egos liegt, stark ein. Manche Menschen haben auch ein prinzipielles Lebensgefühl des „zu kurz Gekommenen".

Woher das kommt, liegt - ganz psychoanalytisch - tief verwurzelt in der Kindheit. Da nicht jeder Mensch in Therapie geht, um dem eigenen Min- derwertigkeitsgefühl auf den Grund zu gehen, wählen viele einen direkten Weg, nämlich ihr menschliches Umfeld zur Erfüllung ihrer Bedürfnisse zu beanspruchen. Oft geht es gar nicht um Materielles, auch wenn es vordergründig so aussieht. Dahinter steht fast immer das Verlangen nach Aufmerksamkeit, Zuwendung oder Nähe. In medizinischen Einrichtungen spitzt sich diese Verhaltensstruktur oft zu, da Leid, Schmerzen und Angst hinzukommen.

In dieser Konstellation kann man als Arzt schonungslos beansprucht werden. Dies stellt eine große Herausforderung dar, besonders dann, wenn wir zeitlich knapp dran sind. Aber: Sich abzuwenden oder den Patienten hinzuhalten bringt wenig, da solche Patienten erfahrungsgemäß immer wieder kommen. Wenn wir uns die o. g. Motive verinnerlichen, dann entsteht daraus Folgendes:

Wir wenden uns dem Menschen wirklich zu, für ca. 10 Minuten. Unter der Voraussetzung, dass wir von Anfang an zu verstehen geben, dass es eine Ausnahme ist. Ihr Praxisablauf lässt so viel Zeit normalerweise nicht zu und dafür warten andere Patienten. Aber Sie tun es gerne! Das kann anfänglich etwas Überwindung kosten, bringt aber enorm viel und kann bei richtig angewandten Kommunikationstechniken den Patienten öffnen und die tatsächlichen Motivationsgründe für seine Penetranz zu Tage fördern. Und Sie haben die Möglichkeit, mehr zu verstehen, z. B. um auch therapeutisch einzugreifen oder weiter $\mathrm{zu}$ verweisen. Einmal richtig hingehört, spart oft viel Zeit und Leid.

Was den Tonfall des Patienten betrifft: Versuchen Sie es doch einmal mit Charme und Humor. Das überrascht Ihr Gegenüber und wird oft direkt gespiegelt.

- Dr. med. A.-Johannes Michel, Lützowstraße 44, D-81245 München 